\title{
Resilienz aus Verwundbarkeit Der Vulnerabilitätsdiskurs als Chance für eine gesellschaftsrelevante Theologie
}

Hildegund Keul

»Köln - Stuttgart - Hamburg. Mehr Sicherheit für unsere Frauen und Töchter!« Mit diesen Worten zog eine rechtspopulistische Partei 2016 in den Landtagswahlkampf von Rheinland-Pfalz. Besonders deutlich erinnerte "Köln" an die kriminellen Ereignisse der Silvesternacht, die heftige Debatten ausgelöst und den Wechsel von einer Willkommenskultur zur Migrationsabwehr eingeläutet haben. In diesem markanten Ereignis zeigt sich, welche Macht die menschliche Verwundbarkeit in gesellschaftlichen und politischen Umbrüchen erlangen kann. Die Angst davor, dass man selbst oder das Eigene, hier "unsere Frauen und Töchter", verwundet werden könnten, ist ein entscheidender Punkt in den Auseinandersetzungen um Migration, Religion und Gender. Wegen der eigenen Verwundbarkeit wird der Ruf nach Sicherheit in den Migrationsdebatten lauter, die Grenzziehungen zwischen Christentum und Islam verschärfen sich, die Genderdebatten kochen hoch.

Migration offenbart sich als Zeichen unserer Zeit, die von Verwundbarkeiten durchzogen ist. Dem kann auch die christliche Theologie nicht ausweichen. Längst sind die Debatten um Migration und Flucht, Vulnerabilität und Sicherheit religionspolitisch bestimmt. Wenn führende Politiker in Osteuropa die Aufnahme muslimischer Migranten infrage stellen und nur Christen aufnehmen wollen, können sie mit Zustimmung auch von katholischer Seite rechnen. Allen voran führt der ungarische Bischof László KissRigó die Bewahrung christlicher Werte ins Feld, um selbst höchst bedrohte Kriegsflüchtlinge aus dem Land auszuschließen. ${ }^{1}$ So wird ein religiöser Sicherungsdiskurs etabliert, der lieber die muslimischen Menschen der Lebensgefahr aussetzt, als die Infragestellung der eigenen religiösen Machtposition zu riskieren. Die klassische "Herodes-Strategie» kommt zum Einsatz: Man schützt das Eigene, indem man Andere derVerwundung preisgibt. ${ }^{2}$

\footnotetext{
1 Vgl.András Máté-Tóth, Ungarn angesichts der Flüchtlinge - Testfall für die Demokratie, in: HerKor 11 (2015), 601-603.

2 Zur Herodes-Strategie vgl. Hildegund Keul, Weihnachten - das Wagnis der Verwundbarkeit, Ostfildern ${ }^{2} 2014,19-25$.
} 
In dieser gesellschaftlichen Situation entsteht eine neue Politik der Angst, die auf die gewaltpotenzierende Macht derVulnerabilität setzt. Dies verleiht jenem Wissenschaftsdiskurs Aktualität, der gezielt "Vulnerabilität« erforscht und der sich seit etwa 30 Jahren entwickelt. Daher stelle ich nach einem genaueren Blick auf die Bedeutung der Vulnerabilität in der gegenwärtigen Situation den interdisziplinären Vulnerabilitätsdiskurs vor. Anschließend gehe ich der Frage nach, welchen Gewinn es für beide Seiten verspricht, wenn die Theologie sich auf Problemstellungen dieses Diskurses einlässt. Dabei kommt aus katholischer Sicht das 2. Vatikanische Konzil ins Spiel, das die Theologie im Duktus seiner Inkarnationslehre in die Risikozonen der Gegenwart schickt. Der Weg der Inkarnation offenbart sich als Weg mitten in die Verwundbarkeit hinein. Hier eröffnet die Theologie eine neue Perspektive, die auch für gesellschaftliche Debatten relevant ist. Bedeutet Verwundbarkeit immer eine Schwächung, die die Resilienz von Menschen und Gruppen verringert? Oder kann aus dem Wagnis der Verwundbarkeit eine neue Stärke wachsen: Resilienz aus Verwundbarkeit?

\section{Sicherheit und die neue Politik der Angst}

Nicht nur tatsächliche Verwundungen, sondern viel mehr noch die Angst davor, verwundet zu werden, ist in turbulenten Zeiten ein entscheidender Machtfaktor. Politische Gruppierungen wissen das zu nutzen, indem sie diese Angst kräftig schüren. »Heute schächten sie Schafe und Rinder, morgen vielleicht schon Christenkinder" so lautet ein Songtext der rechtsextremen Hooligan-Band "Kategorie $\mathrm{C}$ «. Plurale Verwundbarkeiten üben eine unerhörte Macht aus in jenen Herausforderungen, mit denen Europa aufgrund von Krieg, Armut und Migration gegenwärtig konfrontiert ist. Auf der einen Seite stehen jene Menschen, die an den Grenzen Europas auf Einwanderung drängen, um einer lebensgefährlichen Situation zu entkommen. Aber Verwundbarkeit ist nicht nur für die Flüchtlinge ein Thema. Auf der anderen Seite stehen jene Menschen, die sich von der Flucht so Vieler in ihrer eigenen Vulnerabilität angetastet sehen. Noch im Herbst 2015 haben viele Menschen in Deutschland eine große Bereitschaft gezeigt, diese Verwundbarkeit persönlich und gesellschaftlich zu riskieren und die Grenzen durchlässig zu halten. Seit 2016 verschaffen sich Widerstände immer mehr Raum, nochmals verstärkt durch terroristische Anschläge im Herzen Europas. 
In dieser Herausforderung eröffnet die US-amerikanische Philosophin Judith Butler eine entscheidende Perspektive. Ihr Buch Precarious Life (2005) analysiert die komplexen Machtwirkungen, die das Attentat auf das World Trade Center in New York freisetzte. Ihr Ausgangspunkt ist die Feststellung, dass nicht nur die körperliche, sondern auch die soziale Verletzlichkeit das menschliche Leben unausweichlich konstituiert. Butler setzt bei der Trauer um die verlorenen Menschenleben an und stellt die Frage, "was politisch gesehen aus der Trauer anderes entstehen könnte als der Ruf nach Krieg «. ${ }^{3}$ Nach meiner Einschätzung formuliert Butler hiermit eine, wenn nicht die zentrale Frage der Gegenwart. Sind Einzelpersonen, Gruppen und Staaten im Stande, Gewaltspiralen zu unterbrechen? Welche Praktiken können sie entwickeln, um die Machtwirkungen der Gewalt außer Kraft zu setzen? Wie kann aus der erlittenen Destruktion etwas Neues entstehen, das nicht weiteres Leben zerstört, sondern neues Leben schützt, fördert und hervorbringt?

Wunden sind gefährlich, weil sie Leben beschneiden oder töten. Aber die Gefahr von Wunden geht weit über sie selbst hinaus, da sie in der Lage sind, das persönliche und gesellschaftliche, politische und religiöse Leben zu formatieren. Eine Wunde kann unberechenbare Machtwirkungen auslösen, selbst wenn sie verheilt. Denn es bleibt eine Narbe zurück, in der sich die Vulnerabilität verkörpert - im wahrsten Sinn des Wortes. Die Narbe erinnert alltäglich daran, dass man erneut Opfer von Gewalt werden kann. Dies setzt aufwändige Sicherungsmechanismen oder gar Rachefeldzüge in Gang. Denn man hofft, dass man auf diesem Weg resilient wird gegenüber drohenden Gefahren. Wirksam ist also nicht nur die Wunde selbst, sondern die Verwundbarkeit, die sich in ihr verkörpert.

Das Problem gewinnt nochmals an Schärfe, wenn man bedenkt, dass am Anfang einer Sicherungsstrategie nicht einmal eine tatsächlich erfolgte, sondern auch eine nur befürchtete Wunde stehen kann. Mit den Machtwirkungen imaginärer Wunden arbeitet beispielsweise der Rechtspopulismus. Er schürt Ängste vor der angeblichen Gewaltbereitschaft der Flüchtlinge, obwohl diese selbst in erster Linie Opfer von Gewalt sind. Er gibt vor, das christliche Abendland vor seiner Zerstörung zu schützen, indem er selbst Gewaltbereitschaft propagiert und praktiziert. Aber wenn man bedrohte Menschen der Lebensgefahr aussetzt, um christliche Werte zu sichern, so zerstört man die Nächstenliebe und damit einen Grundpfeiler christlichen Glaubens. Eine vermeintliche Schutzstrategie zerstört das, was sie

3 Judith Butler, Gefährdetes Leben. Politische Essays, Frankfurt a.M. 2005, 7. (Original: Precarious Life.The Powers of Mourning andViolence, London/New York 2004). 
zu schützen vorgibt. Politische Gruppierungen schüren die Angst vor Verwundung und wollen so die menschliche Verwundbarkeit als Motor ihres Politkalküls nutzen. Sie sagen: Wer sich verwundbar macht, hat schon verloren. Die Philosophin Martha Nussbaum spricht wegen solcher Politik der Angst und dem Gewaltpotential, das hierin lauert, von der "neuen religiösen Intoleranz«. ${ }^{4}$

In den politischen Debatten zeigt sich ein Spannungsverhältnis von "verwundbar und schwach" oder "resilient und abgesichert«. Meist denkt man, dass sich diese beiden Pole wie eine Waage verhalten: je verwundbarer, desto unsicherer; je abgesicherter, desto weniger verwundbar. Dabei wird übersehen, dass auch Sicherungsstrategien neue Gefahren hervorbringen und damit die Verwundbarkeit erhöhen. Als im März 2015 der Co-Pilot des Germanwings-Fluges 9525 sein Flugzeug mit 150 Menschen zum Absturz brachte, verhinderten die Sicherungen, die das Cockpit abschirmten, dass der Pilot die Tat verhindern konnte. ${ }^{5}$ In der gegenwärtigen Migrationsproblematik ist das Phänomen allerorten zu beobachten, dass Grenzziehungen und die damit verbundenen Ausschließungen Aggressionspotentiale wecken. Sicherungsstrategien allein machen die Grenzen Europas nicht sicher.

In der Migrationsproblematik zeigt sich eine Komplexität pluraler "Verwundbarkeiten«, die eine genaueres Hinschauen erfordern. An dieser Stelle kommt der Vulnerabilitätsdiskurs ins Spiel und die Frage, welche Relevanz die Theologie in diesem Themenfeld erlangt.

\section{Der interdisziplinäre Vulnerabilitätsdiskurs und die fehlende Theologie}

Bislang ist kaum erforscht, wie der Vulnerabilitätsdiskurs genau entstanden ist. Weite Bereiche stehen noch ohne explizite Verbindung da, man weiß nur wenig voneinander. Die Entwicklung eines neuen Diskurses braucht Zeit, bis er überhaupt als eigenständiges Phänomen sichtbar wird und thematisiert werden kann. Eine Metareflexion, die nach inhaltlichen Schwerpunkten, pluralen Methoden und interdisziplinären Verbindungen fragt, beginnt gerade erst. So gibt es

\footnotetext{
4 Vgl. Martha Nussbaum, Die neue religiöse Intoleranz. Ein Ausweg aus der Politik der Angst, Darmstadt 2014 (Original:The New Religious Intolerance. Overcoming the Politics of Fear in an Anxious Age, Cambridge 2012).

5 Ein klassisches Beispiel christlicher Religionskultur sind die Klöster. Ihre Mauern und Tore bieten Schutz - aber sie werden zur Falle, wenn im Inneren ein Feuer ausbricht.
} 
mittlerweile eine erste, noch lückenhafte Übersicht zu "Vulnerabilität in verschiedenen Wissenschaften " ${ }^{6}$. Besonders markant ist, dass die Theologie hier mit keinem Wort erwähnt wird.

Der Vulnerabilitätsdiskurs speist sich aus unterschiedlichen Quellen. Einen ersten Anstoß gab der Wirtschaftsnobelpreisträger Amartya Sen in den achtziger Jahren mit seinen Forschungen zu Hungersnöten, Armut und Gewalt. Er stellte fest, dass einzelne Menschen und gesellschaftliche Gruppen im Hinblick auf bestimmte Gefahren wie Naturkatastrophen in sehr unterschiedlichem Maß verwundbar sind, weil ihre "entitlements", also ihre Verfügungsrechte im Zugriff auf vorhandene Ressourcen, sehr verschieden sind. ${ }^{7}$ Zwar spricht Amartya Sen eher beiläufig von "vulnerability", doch in Auseinandersetzung mit seinem Ansatz etablierte sich der Begriff sehr schnell in Armutsforschung und Entwicklungspolitik ${ }^{8}$, besonders auch bei den UN-Gremien, wo "Armut und Verwundbarkeit» mittlerweile zu den geläufigen Grundbegriffen gehört.

Der Entwicklungsschub in der Armutsforschung wurde von Medizin und Psychologie verstärkt, die es immer schon mit Verwundungen zu tun haben und die nun auf "Verwundbarkeit" eingehen konnten. Sie entwickelten die Gegenüberstellung von "Vulnerabilität und Resilienz«, die in der Forschung derzeit auch in anderen Wissenschaften Fuß fasst; auch hier zeigt sich eine spannende neue Entwicklung, denn Resilienz wird nicht mehr nur im Blick auf Individuen, sondern auch im Blick auf Kulturen, Gesellschaften und Landschaften erforscht. ${ }^{9}$

\footnotetext{
6 Daniel Burghardt u.a., Vulnerabilität in verschiedenen Wissenschaften: Ein Überblick, in: behinderte menschen. Themenheft Verwundbarkeit und Widerstandskraft, 39/2 (2016), 19-31.

7 Vgl. Amartya Sen, Poverty and Famines. An Essay on Entitlement and Deprivation, New York 1981. - Ein Schlüsselereignis war für Sen die Hungersnot von Bengalen im Jahr 1943, bei der etwa drei bis fünf Millionen Menschen verhungerten, obwohl das Land gleichzeitig Lebensmittel exportierte. Es gab nicht zu wenig Lebensmittel, sondern die Hungernden konnten nicht über sie verfügen.

8 Robert Chambers, Vulnerability, Coping and Policy. Editorial Introduction. IDSBulletin April 1989, Volume 20, Issue 2.

9 Die Ausweitung des Begriffs wird besonders deutlich in: Rüdiger Wink (Hg.), Multidisziplinäre Perspektiven der Resilienzforschung (= Studien zur Resilienzforschung 1), Wiesbaden 2016. Zur Debatte um Vulnerabilität und Resilienz in der Sicherheitspolitik vgl. Herfried Münkler/Felix Wassermann, Von strategischer Vulnerabilität zu strategischer Resilienz. Die Herausforderung zukünftiger Sicherheitsforschung und Sicherheitspolitik, in: Lars Gerhold/Jochen Schiller (Hg.), Perspektiven der Sicherheitsforschung. Beiträge aus dem Forschungsforum Öffentliche Sicherheit, Frankfurt a.M. 2012, 77-95.
} 
Die Naturwissenschaften setzen eigene Akzente, indem sie Vulnerabilität messbar machen und besonders in der Katastrophen- und Klimafolgenforschung fokussieren. Mittlerweile ist Vulnerabilität ein Schlüsselbegriff in Medizin und Gesundheitspolitik, Hydrogeologie und Agrarökonomik, philosophischer Ethik und Anthropologie, in Konflikt- und Friedensforschung, Stadtentwicklungs- und Migrationsdebatten oder in den Forschungen zu Resilienz und Glück. Der aus meiner Sicht markanteste Punkt besteht darin, dass "Vulnerabilität« offensichtlich in allen Wissenschaftsbereichen anschlussfähig ist, also nicht nur in den Lebens- und Naturwissenschaften, sondern auch in den Geistes-, Sozial- und sogar in den Ingenieurwissenschaften.

Und die Theologie? Wunden und Leid sind hier ein klassisches Thema und werden beispielsweise in der Kreuzestheologie behandelt. Auch zur Verletzlichkeit gibt es schon länger bemerkenswerte Ansätze. ${ }^{10}$ Neu ist allerdings, dass sich die Theologie mit dem interdisziplinären Diskurs als Ganzem auseinandersetzt. Das war im Jahr 2011 noch nicht der Fall, als meine eigenen Forschungen mich in diese Richtung führten. Mit »Vulnerabilität als Schlüsselbegriff der Theologie « hatte ich zunächst innertheologisch bei der Inkarnation angesetzt. Aber im Oktober 2011 trat die gesellschaftliche Relevanz des Themas ganz neu vor Augen, als öffentlich diskutiert wurde, dass nun sieben Milliarden Menschen auf der Erde leben. ${ }^{11}$ Die breite Debatte, die der damalige Bericht des Bevölkerungsfonds derVereinten Nationen auslöste, lieferte für meine Forschung einen Schlüssel, denn es wurden Fragen öffentlich diskutiert, die um die menschliche Verwundbarkeit kreisen. Wie viele der sieben Milliarden Menschen werden an Hunger, Krankheit und Verelendung sterben? Welchen Migrationsdruck wird die wachsende Weltbevölkerung auf Europa ausüben? Wie können wir uns schützen vor der Gewaltsamkeit, die infolge der ungerechten Verteilung globaler Lebensressourcen ausbrechen kann? Welche religiösen Transformationsprozesse werden die globalen Migrationsströme auslösen?

Mein Interesse war geweckt. Ich wollte wissen, ob und wie das Thema eigentlich in anderen Wissenschaften debattiert wird. Dabei

${ }^{10}$ Ein Klassiker ist zweifellos: Dorothee Sölle, Das Fenster derVerwundbarkeit. Theologisch-politische Texte, Stuttgart 1987. Dagegen verortet Sarah Coakley »Macht in Verwundbarkeit" allein im Spirituellen; vgl. Sarah Coakley, Powers and Submissions. Spirituality, Philosophy and Gender, Oxford 2002 (Dt.: Macht und Unterwerfung. Spiritualität von Frauen zwischen Hingabe und Unterdrückung, Gütersloh 2007).

${ }^{11}$ Ende 2016, also innerhalb von fünf Jahren, ist die Zahl bereits auf 7,5 Milliarden angestiegen. Umso drängender wird die Frage, wie wir mit der Verwundbarkeit der Menschheit umgehen. 
stellte ich fest, dass "vulnerability in sehr diversen Kontexten eine zentrale Rolle spielt und in verschiedenen Wissenschaften aktuell verstärkt diskutiert wird: Es gibt einen »Vulnerabilitätsdiskurs«. In der Klimafolgenforschung ist der Bedarf offensichtlich, denn für die Zukunft der Weltbevölkerung ist es entscheidend, die gegenwärtigen Verwundbarkeiten von Erdbebenzonen, Küstenregionen, Inseln und anderen besonders vulnerablen Landstrichen zu kennen, um eventuell gegensteuern zu können. »IT- und Cyber-Sicherheit«, die die Verwundbarkeiten im System erkennt und abwehrt, ist längst ein globales, auch wissenschaftlich erforschtes Thema. Für die Risikokalkulation der finanzstarken Versicherungsbranche sind die Ingenieurwissenschaften wiederum unverzichtbar, die ihr Augenmerk auf die Verwundbarkeit von Gebäuden und Städten richten.

Der Stand der Forschung in Deutschland spiegelt sich in den Projekten, die von der Deutschen Forschungsgemeinschaft (DFG) zum ThemenfeldVulnerabilität gefördert werden. ${ }^{12}$ Während im Jahr 1999 (dem ersten Jahr der digitalen Erfassung) die Förderung von lediglich zwei Projekten startete, waren es im Jahr 2015 bereits 20 Projekte.

Förderbeginn 2000-2004: 26 Projekte 2005-2009: 47 Projekte 2010-2014: 84 Projekte

Auffällig ist, dass die Theologie hier nicht vertreten ist, obwohl Wunden und Leid zu ihren Kernkompetenzen gehört.

Wie kann sich die Theologie im Diskurs verorten, und was kann sie Neues einbringen? Der Schlüsselbegriff "Inkarnation" bietet dazu einen vielversprechenden Ansatz. Meine Überlegungen hierzu publizierte ich 2012 in "Inkarnation - Gottes Wagnis der Verwundbarkeit « ${ }^{13}$. Erstmals überhaupt wurde hier Vulnerabilität als Schlüsselbegriff interdisziplinärer Forschung in seiner Bedeutung für die Theologie reflektiert. Von "Vulnerabilitätsdiskurs" war bis zu diesem Zeitpunkt auch in anderen Wissenschaften kaum die Rede. Einzelne Forschungsbereiche arbeiteten teilweise zwar sehr gut mit Nachbardisziplinen zusammen, aber der Gesamtdiskurs war nicht im Blick. Seit 2013 wird der neue Diskurs in der Theologie allmählich wahrgenommen und diskutiert, besonders in der Anthropologie und Ethik, aber langsam auch in weiteren Bereichen der Systematischen

${ }^{12}$ Vgl. http://gepris.dfg.de. - Eine tabellarische Aufstellung findet sich auf meiner Homepage www.vulnerabilitätsdiskurs.de.

${ }^{13}$ Hildegund Keul, Inkarnation - Gottes Wagnis derVerwundbarkeit, in:ThQ 192/3 (2012), 216-232. 
Theologie..$^{14}$ Man beginnt zu erforschen, in welchen Feldern er für die Theologie relevant sein könnte und was die Theologie eventuell auch für andere Wissenschaften beizutragen hätte.

\section{Von Wunden zur Vulnerabilität}

Für die christliche Theologie ist der Vulnerabilitätsdiskurs von großem Interesse. Denn auf der einen Seite gehören Verwundungen und Gewalt, Armut und Leid von Beginn an zu ihren Kernthemen. Daher stellt sich die Frage, welche neuen Perspektiven die Theologie in den lange Zeit theologiefreien Diskurs einbringen kann. Darüber hinaus verändert die Neuausrichtung aufVulnerabilität die Theologie und legt hier gänzlich neue Perspektiven frei.Vulnerabilität und Theologie - hier entsteht ein höchst spannender Win-win-Diskurs.

Mit der im 20. Jahrhundert entwickelten Kreuzestheologie (Jürgen Moltmann) sowie der "Neuen Politischen Theologie" (Johann Baptist Metz) bewegte sich die Theologie innerhalb dessen, was ich das "Leidensparadigma" nenne. Der Blick wird hier aufVerwundungen, Gewalt und Leid gerichtet, die von anderen Menschen aus Hass, Missachtung oder Eigennutz ausgeübt werden; die weitere Bedeutung von Vulnerabilität als Zukunftsbegriff war noch nicht im Blick. Herausgefordert von Auschwitz und dem Zweiten Weltkrieg wurde die "Autorität der Leidenden« als "gefährliche Erinnerung« gezielt innertheologisch, besonders in Gottesfragen, zur Geltung gebracht und die Verpflichtung der Kirche zu Compassion und Solidarität eingefordert.

Wunden und Leid sind ein entscheidender Faktor in den prekären Feldern der Macht, mit denen es der Vulnerabilitätsdiskurs zu tun hat. Die große Stärke des Leidensparadigmas besteht darin, dass es mit seiner Sensibilität für die Opfer der Anderen einen ersten wichtigen Aspekt in den Vulnerabilitätsdiskurs einzubringen vermag. Wo werden Anderen Verwundungen zugefügt, weil man sich selbst vor Verwundungen schützen will? Sicherungsstrategien erfordern Opfer, die häufig von anderen Menschen erzwungen werden, weil diese sich nicht wehren können: die Lebensressourcen marginalisierter Länder oder späterer Generationen sowie besonders vulnerable Gruppen, die kein Geld in eigene Sicherungen investieren können. Welche Ressourcen setzt man ein, um eigeneVerwundungen zu ver-

${ }^{14}$ Vgl. das Themenheft »Verletzlichkeit«, ThG 56/3 (2013). Hier wird der Gesamtdiskurs im Editorial von Josef Römelt erläutert, aber in den Einzelbeiträgen spielt er keine tragende Rolle. 
hindern? Die Theologie richtet die Aufmerksamkeit auf die Opfer der Anderen, die das Bemühen um Nicht-Verwundung erzeugt.

Allerdings kann die Theologie diese Perspektive vertreten, ohne sich selbst gravierend zu verändern. Einen eigenen Entwicklungsschub vollzieht sie erst, wenn sie ihre eigenen Debatten in den neuen Diskurs hinein überschreitet. Denn Wunden und Verwundbarkeiten hängen zusammen, sind aber nicht identisch. Man kann sie nicht voneinander trennen, aber man muss sie unterscheiden. Die Thematisierung von Wunden bewegt sich auf der Ebene von Faktizitäten. Verwundbarkeit wiederum ist eine Zukunftskategorie. Man fragt nach möglichen Risiken und entgegenwirkenden Schutzstrategien. ${ }^{15}$ Vulnerabilität bringt eine Potenzialität ins Spiel, eine Möglichkeit in der Zukunft, die bereits in der Gegenwart Wirkungen erzielt.

Hat die Theologie den Blick bisher auf erlittene Wunden fokussiert, so wird der Blick nun auf Verwundbarkeiten hin geweitet. Damit öffnet sich der Blick für jene unerhörte Macht, die die potentielle Gefahr ausübt, verwundet zu werden. Was erschließt der theologische Opferdiskurs mit seinem geschärften Blick für das Leiden der Anderen, wenn er gezielt bei Verwundbarkeiten ansetzt und damit das Leidensparadigma in den Vulnerabilitätsdiskurs überschreitet? Bisher fehlt dem Vulnerabilitätsdiskurs die Theologie mit ihrer Thematisierung von Opfer und Gewalt, Sünde und Erlösung, Gabe und Gegengabe - und vor allem mit ihrer Unterscheidung von victim und sacrifice. Aus christlicher Sicht kann »der verwundbare Gott" neu thematisiert werden. Die Verwundbarkeit rückt dann nämlich nicht erst mit dem Kreuz in den Blick, sondern schon mit der Inkarnation. Indem Gott Mensch wird, macht er sich aus freien Stücken verwundbar - ein höchst verletzliches Kind in der Krippe, dem Matthäusevangelium zufolge von politischer Verfolgung bedroht und von der Flucht nach Ägypten gezeichnet. Inwiefern dieses Wagnis der Verwundbarkeit in heutigen säkularen Kontexten wie Migration und Flucht, Krieg und Frieden Heilsbedeutung erlangt, gilt es eigens zu erforschen. Der Vulnerabilitätsdiskurs erfordert eine Neuformatierung der Inkarnationstheologie. Ihre Entdeckungen gilt es in gesellschaftliche Diskurse sowie in den interreligiösen Diskurs einzubringen. Denn in der Gewaltproblematik von Verwundbarkeit sind Religionen ein entscheidender Faktor.

${ }_{15}$ Als Zukunftskategorie kann Vulnerabilität gravierende, ja sogar verheerende Machtwirkungen ausüben, insbesondere in einer Zeit des "Postfaktischen«. Hierin liegt eine Ursache für den politischen Erfolg rechtspopulistischer Gruppen und Parteien: punktuelle Fakten werden mit enormen Potentialitäten aufgeladen. 


\section{Vom Unsagbaren verletzt}

Bereits 1974 hat der evangelische Theologe Hans Jochen Margull auf die Bedeutung hingewiesen, die das Thema "Verwundbarkeit" für den interreligiösen Dialog hat. ${ }^{16}$ Margull war im Dialog mit dem Islam aktiv und machte die Erfahrung, dass es unweigerlich zur Verwundung kommt, wenn man den Dialog mit einer anderen Religion führt. Verwundbarkeit ist demnach unausweichlich. Wer den Dialog will, vor dem Risiko der Verwundung jedoch zurückscheut, verbaut den Dialog. Margull regte daher an, das Thema inkarnationstheologisch weiterzudenken: Welche Implikationen hat der Glaube an den Gott, der in der Knechtsgestalt verwundbar wird? Margulls Impuls wurde zunächst kaum aufgegriffen und weiter verfolgt. Heute aber kann die christliche Theologie in Europa dem Islam nicht mehr ausweichen, da dieser in alltäglichen Lebenswelten präsent ist und sich langsam auch an den Universitäten etabliert. Dabei ist der gravierende Verlust an Macht und Einfluss, den das Christentum in den letzten Jahrzehnten erfährt, als Verwundung präsent. Sie übt ihre Macht aus, wo Menschen oder Institutionen die christlichen Werte schützen wollen, indem sie islamische Perspektiven strikt außen vor halten. Auf Säkularisierung und die Pluralisierung der Religionsdiskurse reagieren sie nicht mit Öffnung und Dialog, sondern mit Sicherungsstrategien: Werte vor dem Verfall schützen, Traditionen vor ihrem Verschwinden sichern, Heilige Texte und Rituale vor Verletzung durch den Zeitgeist bewahren. Auf die wachsende Heterogenität reagiert man mit verstärktem Homogenisierungsdruck, der über verletzende Ausschließungen funktioniert.

Michel de Certeau, der sich als Theologe und Kulturtheoretiker an der Schnittstelle von theologischen Sicherungsdiskursen und säkularen Verwundbarkeiten bewegte, analysierte solche Problemlagen. Er wandte sich gegen die Theologie im Format des Antimodernismus, die er folgendermaßen beschreibt: „Einst stellte eine Kirche einen Boden bereit, das heißt ein fest umrissenes Terrain, innerhalb dessen man die soziale und kulturelle Garantie hatte, dass man auf dem Acker der Wahrheit wohnte. ${ }^{17}{ }^{\mathrm{U}} \mathrm{Um}$ den Acker der Wahrheit zu bewahren, setzt man auf Homogenität und wehrt alles Heterogene $\mathrm{ab}$. Um die eigene Institution vor Verwundung zu schützen, verwundet man Andere.

${ }^{16}$ Hans Jochen Margull, Verwundbarkeit. Bemerkungen zum Dialog, in: Evangelische Theologie 34 (1974), 410-420.

${ }^{17}$ Michel de Certeau, GlaubensSchwachheit, Stuttgart 2009 (ReligionsKulturen 2), 243. 
Solche Sicherungsmechanismen, die Homogenität erzwingen wollen, werden auch heute gesellschaftlich wie kirchlich installiert. Antimodernismus ist nicht nur eine historische Epoche, sondern eine Haltung und ein Verhalten dem Fremden, Nicht-Kirchlichen gegenüber. Setzt die Kirche allein auf die Strategie der Ausschließung, so wird sie zu einer Art Hochsicherheitstrakt. Mauern, Tore und Wächter unterbinden die Einflüsse von außen und nach außen. Theologisch bewegt man sich im Inneren, d.h. ausschließlich in Fragen, die man aus der Tradition bereits kennt. Der Vorteil des so erzeugten geschlossenen Diskurses liegt darin, dass er zunächst relativ unverwundbar macht. Man kennt sich aus in den eigenen Argumenten, so dass man Anfragen und Angriffen leichtfüßig standhält. Die Theologie genügt sich selbst, sie braucht keine anderen Konfessionen, Religionen oder Kulturen. Allerdings scheitern solche Diskurse an einer Wirklichkeit, die gar nicht daran denkt, sich der propagierten Homogenität zu unterwerfen. Faktisch ereignet sich eine Heterogenität, die am postulierten Acker der Wahrheit nagt. Sie erzeugt Risse und Brüche und dringt beharrlich in das Innere der Kirche ein. Sie wird zu einer Realität, die sich nicht länger verschweigen lässt.

In der katholischen Kirche führte dieser Prozess zum 2. Vatikanischen Konzil. Man musste erkennen, dass die Kirche mit ihren geschlossenen Grenzen zwar nach außen abgesichert war, aber zugleich ihre gesellschaftliche Relevanz verlor - ganz zu schweigen von den inhumanen Opfern, die ihre Ausschließungen produzierte. Das Konzil öffnete den Hochsicherheitstrakt für den Dialog mit den Religionen und der Welt von heute. Solche Öffnungen aber sind riskant, denn sie machen verwundbar. Plötzlich steht man vor Fragen, auf die man keine Antwort parat hat. Andere verweisen auf etwas im eigenen Glauben, das man selbst (noch) nicht sagen kann. "So wird, auf tausenderlei Weisen, das Aussagbare unablässig von etwas Unsagbarem verletzt«, sagt Certeau. ${ }^{18}$ Man ist von etwas Fremdem gezeichnet und trägt Blessuren davon. Unerhörtes macht sich in vertrauten diskursiven Ordnungen als Verletzung bemerkbar. Damit wird der eigene Glaube aus den Feldern der Gewissheit in Ungewissheit geführt, aus einer früheren Stärke in eine gegenwärtige Schwäche, aus einer Haltung der Unangreifbarkeit in das Wagnis derVerwundbarkeit.

${ }^{18}$ Michel de Certeau, Mystische Fabel. 16. bis 17. Jahrhundert, Berlin 2010, 123. 


\section{Theologie in den Risikozonen der Gegenwart}

Christologisch gesehen ist dieser Weg in die Verwundbarkeiten der Welt hinein unausweichlich. Denn es ist die Bewegung der Inkarnation, der »Fleischwerdung«: wie Gott sich mit der Menschwerdung in einer gewagten Gabe seiner selbst freiwillig verwundbar macht, so stellt sich auch die Theologie den körperlichen, sozialen und religiösen Verwundbarkeiten des menschlichen Lebens. Wenn sie sich nicht in einem Hochsicherheitstrakt verschanzen will, der mehr und mehr an Bedeutung verliert, so bleibt ihr nur, dieses Wagnis einzugehen. Sie tritt aus der Homogenität der einen Religion heraus und stellt sich nicht nur der Pluralität der Religionen und ihrer säkularen Bestreitung. Vielmehr ist sie herausgefordert, den Risikozonen der Gegenwart nicht auszuweichen, wo Vulnerabilität ein entscheidendes Thema ist: in die Migrationsbewegungen mit ihren Todesstreifen und Hoffnungsorten, in Kriegsgebiete mit ihren Zonen totaler Kontrolle, in die Megastädte mit ihren Arrival Cities. Die christliche Theologie ist herausgefordert, eigene Perspektiven in säkulare Problemlagen derVulnerabilität einzubringen. Damit macht sie sich angreifbar. Sie kann nicht mehr einen Bestätigungsdiskurs verfolgen, sondern muss auch in dem befragbar sein, was sie nicht sagen kann. $\mathrm{Ob}$ sie bereit ist, dies tatsächlich zu riskieren, wird darüber entscheiden, ob sie neue Gesellschaftsrelevanz zu erlangen vermag.

Der Vulnerabilitätsdiskurs fordert zu einer Inkarnationstheologie heraus, die säkulare Problemlagen der Vulnerabilität aus christlicher Perspektive erschließt. Die Besonderheit liegt darin, dass das Christentum der Inkarnation, also dem Weg in die Verwundbarkeit hinein, Heilsbedeutung zuspricht. Dies widerspricht einem großen Teil der Vulnerabilitätsforschung, wo ein binärer Code von "vulnerabel, unsicher, gefährdet, schwach, angreifbar" versus "resilient, sicher, stark, belastbar, geschützt« herrscht. Die Ingenieur- und Naturwissenschaften betreiben die Vulnerabilitätsforschung als Schwachstellenanalyse. Widerspruch gegenüber dem binären Code kommt vereinzelt aus der Psychologie sowie der Politikwissenschaft. ${ }^{19}$ Theologisch braucht es einen Ansatz, der auch die kreativen, resilienzfördernden Machtwirkungen von Vulnerabilität analysierbar macht. Dies ist im Blick auf die aktuellen Debatten um Migration, Flucht und religionspolitischer Gewalt besonders relevant. Um ein

${ }^{19}$ Die Psychologin Brené Brown erzielt mit ihren Büchern zu Verletzlichkeit Bestseller-Erfolge; vgl. Brené Brown, Verletzlichkeit macht stark. Wie wir unsere Schutzmechanismen aufgeben und innerlich reich werden, München 2013. (Original: Daring Greatly, New York 2012). 
humanes Leben zu führen, genügt Selbstschutz nicht, denn er erfordert immer höhere Mauern, mächtigere Grenzanlagen und schärfere Waffen. Inwiefern sind auch heute Menschen, Gruppen oder gar Staaten bereit, den Weg freiwilliger Verwundbarkeit zu gehen, um gefährdetes Leben zu schützen?

\section{Resilienz aus Verwundbarkeit}

Sich selbst, die eigene Familie, Kultur oder Religion schützen zu wollen, ist legitim, häufig sogar lebensnotwendig. In unserer Gesellschaft werden wir ständig daran erinnert, z.B. von Versicherungen, politischen Parteien, aber auch von lautstarken Demonstrationen. Wer sich nicht vorVerwundungen schützt, wird das schnell mit dem Leben bezahlen. Aber welche Risiken erzeugt der Selbstschutz, welche Opfer erfordert er? Und wer hat diese Risiken zu tragen und diese Opfer zu bringen? Selbstschutz durch Grenzziehung birgt ein Gewaltpotential, das häufig verdeckt ist, weil das Wort "Schutz« im Deutschen einen durch und durch positiven Klang hat. Aber das, was als Selbstschutz gedacht war, richtet sich schnell wie ein Bumerang auch gegen das Eigene.

Das Problem liegt im binären Code "entweder vulnerabel und angreifbar oder resilient und abgesichert", der scheinbar als Nullsummenspiel funktioniert: je mehr Sicherungen man einführt, desto weniger vulnerabel verspricht ein System zu sein. Der binäre Code funktioniert jedoch nicht. Er ist viel zu einfach, um die komplexen Machtwirkungen pluraler Verwundbarkeiten zu erfassen. Schlimmstenfalls mündet er in dem vergeblichen Versuch, sich selbst durch immer mehr Gewalt gegen Andere unverwundbar zu machen.

Auf der Suche nach einem Dritten, das den binären Code durchbricht und überwindet, kommt die neue Resilienzforschung der Theologie ins Spiel. Welche Chancen für einen resilienzfördernden Umgang mit Wunden und Verwundbarkeiten kann sie erschließen? Diese Fragestellung eröffnet die Theologie im Bayerischen Forschungsverbund "Fit for Change.${ }^{20} "$ Resilienz bezeichnet hier die Fähigkeit, Krisen zu bewältigen und durch Rückgriff auf vielfältige Ressourcen problemlösende Entwicklungen anzustoßen. Bezeichnenderweise ist es die Theologie, die feststellt: "Resilienz meint nicht Unverletzlichkeit, sondern das Glück gelingenden Lebens in-

${ }^{20}$ Vgl. das Themenheft »Resilienz« der MünchnerTheologischen Zeitschrift MThZ 3 (2016). Martin Schneider und Markus Wolf gehen der Frage nach, inwiefern Glaube, Liebe und Hoffnung Resilienzfaktoren sind (vgl. ebd., 195-208). 
mitten aller Verwundungen und Unvollkommenheiten. ${ }^{21}{ }^{2}$ Wie sich Verwundbarkeit und Resilienz zueinander verhalten, wird damit zu einer Schlüsselfrage.

Etymologisch gesehen hat der Begriff »Resilienz« den Vorteil, dass er bei einerVerletzung ansetzt: ursprünglich bezeichnet er die Fähigkeit elastischen Materials, nach starkerVerformung (=Verletzung) in den Ausgangszustand zurückzukehren. Resilienz hat es also immer schon mit Verwundungen zu tun. Allerdings wird im säkularen Vulnerabilitätsdiskurs Verwundbarkeit meist als etwas erforscht, das es zu vermeiden oder zu verringern gilt. Ingenieurwissenschaften wollen die Vulnerabilität von Gebäuden reduzieren, Medizin die Krankheitsrisiken minimieren, Ökologie und Geografie die Verwundbarkeit von Lebewesen und Landschaften verringern. Verwundbarkeit bedeutet Störung, Verlust, Versagen, Gefahr, Schädigung, Bedrohung. Eine aktuelle Studie zur Resilienzforschung definiert Vulnerabilität »im Sinne einer andauernden Gefährdung und Beeinträchtigung von Wohlbefinden und Lebenschancen ${ }^{22}$ Aber diese einseitige Sicht von Vulnerabilität erhält Risse. So weist die Psychologin Insa Fooken darauf hin, dass es dann »keine (gleichzeitige) Ausprägung von sowohl Vulnerabilität als auch Resilienz geben" dürfte, ${ }^{23}$ was aber faktisch der Fall ist. Empirisch lassen sich "scheinbar paradoxe Zusammenhänge ${ }^{24}$ zwischen Vulnerabilität und Resilienz feststellen. Das Bild von der Waage trifft die Sachlage nicht.

Dieser Befund ist für die Theologie sehr interessant. Wenn erhöhte Vulnerabilität nicht automatisch zum Absinken der Resilienz führt, stellt sich die Frage, ob es eine Form von Vulnerabilität gibt, die Resilienz steigert. Muss Vulnerabilität wirklich immer zur Schwächung führen? Immerhin entsteht Verwundbarkeit immer dort, wo man berührbar ist, bereit zur Kommunikation mit Anderen, offen zum Austausch mit Fremdem. Das Ziel der Resilienzforschung, Verwundbarkeiten zu verringern, hat selbstverständlich in weiten Bereichen seine Berechtigung. Dennoch wird hier ein zentraler Aspekt im dynamischen Machtgefüge pluraler Verwundbarkeiten vernachlässigt. Denn Menschen, Staaten und Religionen versuchen gar nicht immer, eigene Verwundungen zu verhindern. Vielmehr sind sie überaus bereit, ihre eigene Verwundbarkeit zu riskieren

${ }^{21}$ Ebd., 184; in Fußnote 8 heben die Herausgeber des Themenhefts "Resilienz« hervor, dass alle Beiträge im Heft sich darin einig sind.

${ }^{22}$ Insa Fooken, Psychologische Perspektiven der Resilienzforschung, in: Rüdiger Wink (Hg.), Multidisziplinäre Perspektiven der Resilienzforschung (= Studien zur Resilienzforschung 1). Wiesbaden 2016, 15.

${ }^{23}$ Ebd., 16.

${ }^{24}$ Ebd. 
und Opfer zu bringen, wenn es um etwas geht, das ihnen heilig ist: Frauen bringen Kinder zur Welt, obwohl allein schon die Geburt schmerzlich ist und die Erziehung die finanzielle Absicherung gefährdet; Religionen zetteln Selbstmordattentate und "Heilige Kriege« an, obwohl sie damit Anhänger verlieren und große Risiken eingehen; Staaten öffnen ihre Grenzen und nehmen Flüchtlinge auf, obwohl dies Unsicherheiten aller Art schafft. Daher stellt sich die Frage, ob Verwundbarkeit tatsächlich automatisch schwächt und gefährdet, oder ob und unter welchen Umständen aus Verwundbarkeit Resilienz wachsen kann.

Der christlichen Theologie ist der Gedanke vertraut, freiwillig eigene Verwundbarkeit zu riskieren. Sie vertritt den Glauben an einen Gott, der als Kind zur Welt kommt und sich der Verwundbarkeit menschlichen Lebens aussetzt. Christliche Berufung bedeutet, sich selbst für diesen Weg der Inkarnation zu entscheiden. Dabei geht es nicht darum, Leiden zu glorifizieren.Vielmehr bringt die Theologie in Herausforderungen derVulnerabilität, die häufig - beispielsweise in der rigorosen Migrationsabwehr - Opfer von Anderen verlangen und Gewalt potenzieren, eine andere Macht ins Spiel. Der Apostel Paulus hat diese Macht entdeckt und zur Sprache gebracht, als er sich vor Gott über eine Verwundung, den berühmten Stachel im Fleisch, beklagte. Er hörte die Antwort: "Lass dir an meiner Gnade genügen; denn meine Kraft ist in den Schwachen mächtig." (2 Kor 12,9) Diese Macht, die als Gnade ganz anders funktioniert als mit Mauern, Grenzen und Waffen, diese Andersmacht aus Verwundbarkeit ist dort am Werk, wo Menschen sich hingebungsvoll einsetzen für ihre Mitmenschen, die in Not geraten sind. Wenn man die eigene Verwundbarkeit riskiert, um das Leben Anderer zu schützen und zu fördern, so kann in diesem Wagnis Resilienz entstehen. Man gewinnt Stärke, weil man sich für gefährdetes Leben einsetzt, sich für Gerechtigkeit engagiert oder im Umweltschutz aktiv ist.Vulnerabilität und Resilienz sind dann kein sich wechselseitig ausschließender Gegensatz mehr, sondern hier wächst Resilienz aus Verwundbarkeit. Sie ist in der Lage, Leben zu eröffnen, zu schützen und damit zu sichern. Hier handelt es sich um eine Form der Resilienz, die den Gewaltspiralen der Verwundbarkeit widersteht. Sie ist im Leben jener Menschen und Gemeinschaften am Werk, die in der Gewaltsamkeit menschlicherVulnerabilität Hingabe wagen, um gefährdetes Leben zu schützen.

Wenn man in Zukunft die beiden Diskurse um Vulnerabilität und Resilienz stärker zusammenführt, so hat die Theologie überraschende Perspektiven einzubringen. Dabei wird die Unterscheidung von 
victim (eine Verwundung erleiden) und sacrifice (freiwillig Verwundbarkeit riskieren) eine zentrale Rolle spielen. ${ }^{25}$ In der Verwundbarkeit ist nicht nur jene unerhörte Macht am Werk, die Opfer von Anderen verlangt und damit Gewalt unendlich potenziert. Vielmehr gibt es eine Andersmacht, die dem entgegenwirkt und aus Verwundbarkeit Resilienz entstehen lässt. Im Spannungsfeld von Verwundbarkeit und Sicherheit, Vulnerabilität und Resilienz ist es auch für eine Gesellschaft entscheidend, ob sie darauf setzt, dass das Wagnis eigener Verwundbarkeit zur Sicherung des Lebens beitragen kann. Wenn Vulnerabilität und Resilienz sich tatsächlich gegenseitig ausschließen würden, dann hätte tatsächlich schon verloren, wer sich verwundbar macht. Der Fehler liegt im Dualismus von Vulnerabilität und Resilienz. Die Geschichte des Christentums und der Menschheit insgesamt ist voller Beispiele dafür, dass aus dem Wagnis eigener Verwundbarkeit Resilienz erwachsen und damit eine andere Art von Sicherheit entstehen kann. Gerade die Zerbrechlichkeit des Lebens erfordert Menschen, die sich in Liebe, Zuneigung und Barmherzigkeit verletzlich machen. Sie gehen gestärkt aus diesem Wagnis hervor. Aus Schwachheit wird Stärke, aus dem Wagnis der Verwundbarkeit wächst Resilienz.

- Hildegund Keul, apl. Professur für Fundamentaltheologie und vergleichende Religionswissenschaft an der Universität Würzburg; Leitung der Research Group »Vulnerabilität, Sicherheit und Resilienz«. Forschungsschwerpunkt: Vulnerabilität als Schlüsselbegriff in Theologie und interdisziplinärer Wissenschaft: www.vulnerabilitätsdiskurs.de

${ }^{25}$ Vgl. Hildegund Keul, Vulnerabilität und Resilienz - christlich-theologische Perspektiven, in: MThZ 67 (2016), Themenheft Resilienz, 224-233. 